\title{
Multivariate analysis and optimal configuration of wind- photovoltaic complementary power generation system
}

\author{
WANG Guangwei ${ }^{1 *}$, SU Ziqin ${ }^{1}$, LI Shixin ${ }^{1}$, CHEN Tai $^{1}$ \\ ${ }^{1}$ School of Electronic Engineering, Tianjin University of Technology and Education, Tianjin 300222, China
}

\begin{abstract}
Advantages of wind-solar complementary power generation system to utilize solar and wind energy in the aspect of resource and technical economy have been reviewed tersely. Convenience of entering and exiting generating equipment and load from DC as well as AC bus are interpreted briefly. The factors that affect the electrical power output of the system were analyzed and studied. Based on the law of energy conservation, the energetic matching algorithm was proposed which forms the foundation of optimal configuration of system. Finally, the intelligent control and on-line monitoring of wind-solar complementary power generation system were discussed.
\end{abstract}

\section{Introduction}

Wind and solar energy have some shortcomings such as randomness, instability and high cost of power generation. Wind-solar complementary power generation system is the combination of their advantages. The system converts solar and wind energy into electric energy for load and conducts long-distance transmission, a hot topic in the field of renewable green energy, which integrates energetic conversion, storage and grid connection technology. Wind-solar complementary power generation system has such advantages as no pollution, low noise and high reliability. At present, the technology of solar and wind energy complementary power generation is becoming more mature, therefore a number of power stations have been built in some coast, grassland and

Gobi desert in China, with unprecedented speed of popularization. However, unreasonable tracking control strategies and mismatch between generation and energetic consumption is a key problem, which reduces economical and technical benefits enormously ${ }^{[1]}$.

The wind-solar complementary power generation system is composed of solar photovoltaic array, wind turbine generator sets (WTGS), intelligent controller, valve-controlled sealed lead-acid battery pack $^{[2]}$ (or lithium ion battery module, which is discussed later in this paper), inverter, load and other parts. Figure 1 is the structural composition diagram of off-grid wind-solar complementary power generation system.

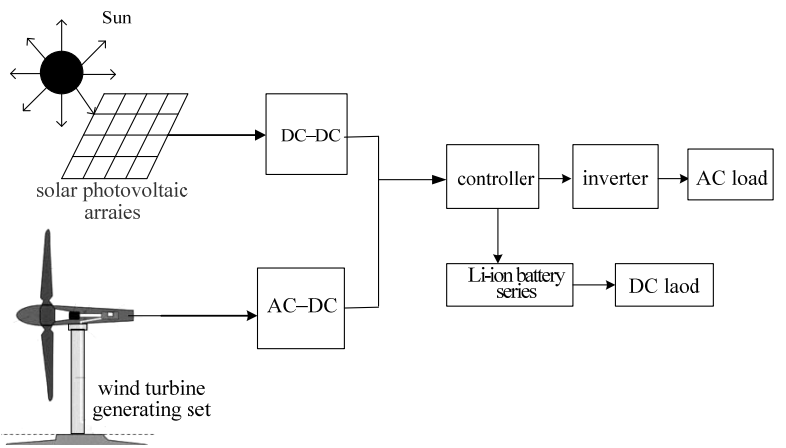

Fig 1. Structural diagram of off-grid wind-solar complementary power generation system

WTGS is usually installed in windy areas, and brake device ensures to reduce blade speed when wind speed is too high. The output electrical power of WTGS is related to wind speed, blade length and shape. If wind speed is too high, a kind of light materials named phenolic epoxy vinyl ester resin carbon fiber composites used in blade with high strength, is a better one to withstand strong winds with bending strength of $1500 \sim 1900 \mathrm{MPa}$ and tensile strength close to $700 \mathrm{MPa}^{[3]}$. Random variation of wind speed and direction leads to fluctuating in output electric energy of wind power system. There are two solutions. One is to use lithium ion battery module+supercapacitor to store more electric energy; the other is to run wind power and photovoltaic, Diesel power and thermal power in complementary operation mode, also known as multienergy complementary system.

In recent years, with rapid technological progress of wind-solar complementary power generation, some large tool software packages have been adopted to simulate performance of system. The optimal parameters are obtained by comparing performance and cost of power supply in different configurations. Hybrid 2 is an excellent 
software package. According to topological structure of the system, input parameters, load characteristics, wind speed, solar radiation intensity and other data, simulation operation result of 8760 hours in one year was obtained, which was in good agreement with the actual situation. However, Hybrid 2 is just simulation software with strong function, better than Matlab/Simulink module, but functions of optimized design are not enough, and it is expensive, professional, and inconvenient to use for users.

Mainly, there are two methods to optimize the design of wind-solar complementary power generation system: one is power matching, that is, in condition of different solar irradiance and wind speed, the total power of photovoltaic array as well as WTGS is not less than that of load, which is used for optimal control of the system; the other is energetic matching one, in which the sum of power generation of photovoltaic array and WTGS is not less than the total power consumption of load (including the system energy consumption), which is mainly used to optimize design, and will be discussed later in this article. A core index of optimization of wind-solar complementary power generation system is power shortage rate, whose definition is the ratio of load power shortage to total load demand, a variable value. The smaller the value, the better ${ }^{[4]}$.

\section{Overall analyses}

\subsection{Analysis of resource}

Some areas in western China are sparsely populated, where relatively small electricity power is needed, and long-distance power transmission is uneconomical. It is a better choice to generate electricity locally and build micro-grids. Wind-solar complementary power generation is a good project of stable power supply. Wind energy refers to kinetic energy resulting from air flow, which is directly related to temperature difference caused by different absorption coefficients of solar energy on surface of different forms (such as sandy soil, vegetation and water).Solar and wind energy are complementary in time and place. During the daytime, sunlight is strong except rainy day, while wind speed is low; at night, light is weak, but the surface temperature difference of object becomes larger and wind may be stronger. In sunny summer, sunlight is generally strong and wind is weak. In winter, when it is sunny, sunlight is weak while wind may be strong. Solar and wind energy have a good match in terms of resources, so wind-solar complementary power generation system is a better scheme to utilize solar and wind energy simultaneously. Research results show that systematic stability has been improved significantly under optimal ratio of wind and sunlight resources ${ }^{[5]}$.

\subsection{Technical and economical analysis}

The photovoltaic power generation system converts solar energy into electricity, charging lithium-ion battery modules through controller and supplying power to AC load through inverter. Advantages are high reliability, low cost of operation and maintenance, long service life, while the main disadvantage is that the initial investment of the system is high relatively.

The WTGS converts wind energy into electricity, after rectification, filtering and voltage regulation, supplying power to load through an inverter. Advantages are higher generating efficiency, low cost of operation and maintenance. Disadvantages are low reliability and poor stability. In addition, the common weakness of wind power and photovoltaic system is the uncertainty of resources which leads to mismatch between power generation and electrical load. Wind power and photovoltaic generation system can supply electric energy stably through energetic storage in lithium ion battery module, but daily power output is affected greatly by weather conditions, which may give rise to lack of electricity and shorten service life period. Optimizing design of system capacity according to power load and resource conditions ensures reliable power supply and reduces cost. No matter what meteorological condition and electricity requirements are, there is always an optimal solution to meet the requirements of users. At present, the main bottleneck of renovating and expanding wind-solar complementary power generation project is the optimization of system configuration. According to research dada, the overall performance of wind-solar complementary power generation system is better than that of wind farms with the same installed capacity. It is of certain practical engineering significance to expand windsolar complementary system in completed wind farms to improve utilization rate of green renewable energy ${ }^{[6]}$.

Speed limiting of wind motor by hydraulic brake can improve stability obviously, reduce impact of wind speed fluctuation on components and improve efficiency of electrical output ${ }^{[7]}$. In high winds, it is common to limit speed through controlling blades mechanically. Mechanical speed limit is characterized by dynamic support of machine head or a certain part. In wind tunnel test, the effects of speed limit are good. However, under natural conditions, due to variation of wind speed and direction is too complex (mainly turbulent), the vibration of support parts of blade and wear of moving parts, which may lead to damage WTGS. At present, only three moving parts are retained. One is the wind blade driving main rotor shaft of the generator; the other is the tail fin driving the head yaw; and the third is crosswind yaw system which can protect the wind motor from burning down due to excessive wind speed ${ }^{[8]}$.The first two moving parts are indispensable. In practice, failure rate of these two moving parts is not too high; meanwhile the damage probability of speed limit institutions is high. In view of shortcomings of mechanical speed limit, magnetoelectric speed limit is proposed originally. By theoretical analysis, when rotation speed of blade is too fast, a reverse magnetic resistance will generate through consumption of electricity, increasing rotor damping, lowering blades rotating speed. The mechanical speed limiting institution is abandoned; only two moving parts necessary for the wind motor are kept, ensuring safe and reliable operation of wind turbine for a long time of period. 


\section{Bus structure of wind-solar complementary power system}

It can be divided into DC and AC bus structure and they play an important role in wind-solar complementary power generation system.

\subsection{Structure of DC bus}

Figure 2 shows the distributed system of DC bus control. The controlling algorithm of this system is simple, the cost is low, and it is easy to expand. For off-grid power supply system, the capacity can be expanded artificially.

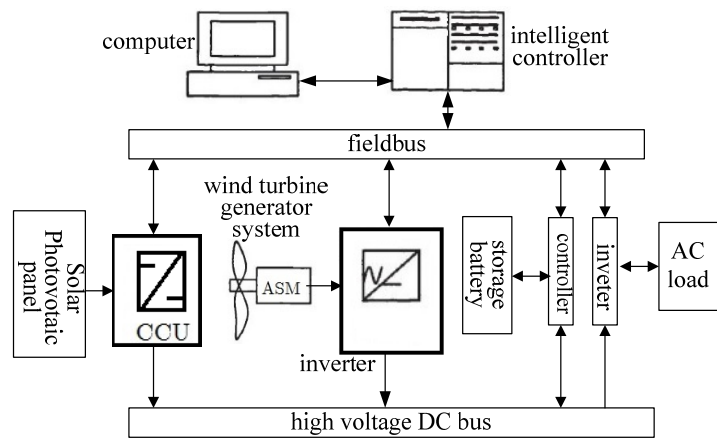

Fig 2. Structure diagram of distributed DC bus in windsolar complementary power generation system

The main advantages of this system are:

(1) Only controlling the bus voltage can meet performance requirements, also the controlling algorithm is relatively simple.

(2) Rectifying part can be omitted, the system cost is reduced and easy to be popularized.

(3) It is easy for distributed DC bus to expand and can meet requirements of the increase and decrease of equipment of electricity consumption or power generation. With DC bus, the energy storage unit is divided into storage units with long term and short term. The lithiumion battery module is a long-term energy storage device. Short-term energy storage unit can compensate for bus voltage fluctuation caused by change of wind speed, illumination of the Sun and load. In order to improve practicability, the DC bus is extended. For example, to ensure normal operation of important electrical equipment, Diesel power generator set may be installed on DC bus, and electric energy is allocated uniformly through intelligent controller. DC/DC converter supplies power to DC equipment, without rectifier module and avoiding harmonic environmental pollution.

\subsection{Structure of $A C$ bus}

All kinds of generating equipment or loads connect to the same AC bus. When load or power generation equipment is added, systematic capacity can also be expanded at will. The structure of AC bus distributed system of wind-solar complementary power supply is shown in Figure 3.

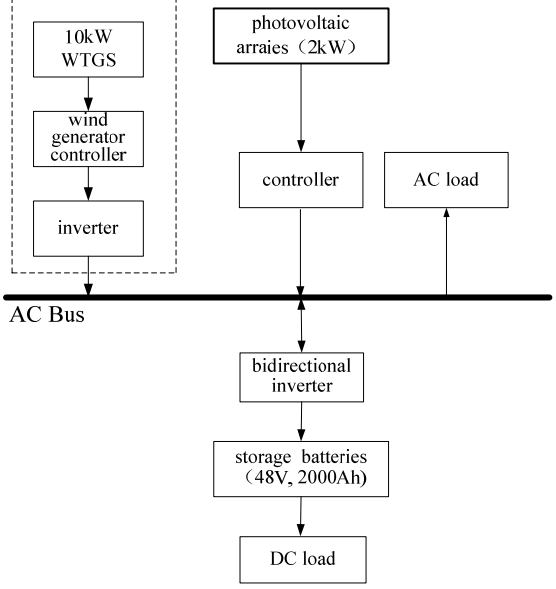

Fig 3. Block diagram of $\mathrm{AC}$ bus in wind-solar complementary power generation system

In Figure 3, a 10kW WTGS, a blade controller and an inverter constitute a unit. The $2 \mathrm{~kW}$ photovoltaic array outputs direct current, which is converted into alternating current through inverter and connected to bus. AC bus is connected to AC load through a switching controller, or connected to power grid directly. Bidirectional inverter converts alternating current in $\mathrm{AC}$ bus into direct current so as to charge lithium-ion battery modules and store electrical energy.

\section{Optimal configuration of wind-solar complementary power generation system}

\subsection{Factors to be considered}

\subsubsection{Solar and wind energy resources}

According to status of solar and wind energy, the capacity of photovoltaic panels and WTGS is determined. Confirming electricity load and considering capacity coefficient, the number of photovoltaic panels and the capacity of wind driven generator is decided. For gridconnected wind-solar complementary systems, the objective of optimization should be to minimize impact on system as well as power grid and maximize environmental protection and economic benefits ${ }^{[9]}$. Selection of system capacity is stipulated in the national standard "Technical Conditions for Off-grid Household Wind-solar Complementary Power Generation System (GB/T19115.1-2003)". In short, a wind-solar complementary power generation system is reflected in reasonable resource allocation, feasible technical scheme and cost-efficient performance, which ensures its popularization and application.

\subsubsection{Algorithm of optimal systematic configuration}

In order to provide stable electric energy for users, matching between the maximum power load and power generation should be considered in detail. The maximum 
power load is the basis for capacity selection of inverter, and average daily power generation is the foundation for determining capacity of WTGS, photovoltaic panel and lithium-ion battery modules. When there is a tracking system in photovoltaic power generation system (PV), the maximum output power needs to be considered too. For systematic design, modeling at first, using computer program to simulate, analyzing simulation results, the optimal configuration parameters are gotten. Considering the law of conservation of energy, the following equation should be satisfied in any period of time:

$$
\sum_{i} C_{i}^{(1)} \Delta_{i} \Delta_{i}+\sum_{j} C_{j}^{(2)} \Delta P_{j} \Delta_{j}=\max \left(\sum_{k} C_{k}^{(3)} \Delta_{k} \Delta_{k}\right)+\Delta E(t)
$$

On the left side of formula (1), items 1 and 2 are photovoltaic and wind power generation capacity respectively. Items 1 and 2 on the right are peak electricity consumption (including energy consumption of the equipment) and stored energy, respectively. $C_{i, j, k}{ }^{(m)}$ is the systematic correlation coefficient $, m=1,2,3$, and $i, j, k$ just take natural numbers, $\Delta E(t)$ signifies the amount of energetic storage, a negative value indicates power loss; While a positive value can represent the amount of energy stored in the battery/ultracapacitor, the amount needed to heat water, the amount needed to lift water to higher places, or the amount needed to make hydrogen gas, etc.

Photovoltaic generation power depends mainly on the total solar radiation intensity $I(t)$, while the wind generation power mainly depends on wind speed $v(t)$. If energy storage is temporarily omitted, and the influence of temperature variation is small. Therefore, Equation (1) is simplified as follows,

$\sum_{i} C_{i}^{(1)} \Delta P_{i}\left[I_{i}(t)\right] \Delta \Delta_{i}+\sum_{j} C_{j}^{(2)} \Delta P_{j}\left[v_{j}(t)\right] \Delta t_{j}=\max \left(\sum_{k} C_{k}^{(3)} \Delta P_{k} \Delta t_{k}\right)$

$$
\text { In Equation (2) , } C_{i}^{(1)} \Delta P_{i}\left[I_{i}(t)\right] \Delta t_{i},
$$
$C_{j}^{(2)} \Delta P_{j}\left[v_{j}(t)\right] \Delta t_{j}$ are electric energy generated by photovoltaic power system and wind turbine respectively; $C_{k}{ }^{(3)} \Delta P_{k} \Delta t_{k}$ is electrical energy consumed by load during the corresponding period of time $\Delta t_{k}$. When approximating, the functional relationship between photovoltaic power and sunlight intensity is used to calculate $\sum_{i} C_{i}^{(1)} \Delta P_{i}\left[I_{i}(t)\right] \Delta t_{i}$ by integral, it will not be repeated here. Considering weather rain or shine, rain or snow, night and temperature change , $\sum_{i} C_{i}^{(1)} \Delta P_{i}\left[I_{i}(t)\right] \Delta t_{i}$ can be calculated by NWP Kalman filter ${ }^{[10]}$, Compared with the recorded PV historical data, it is considered to be accurate under the condition of good consistency. In a certain range, output power of WTGS, $\Delta P_{j}\left[v_{j}(t)\right]$ increases with the increase of wind speed $v_{j}(t)$, but change of wind speed and direction is far more complex, and disturbance causes fluctuation of wind power output. It is assumed that the natural wind speed satisfies the Wimbledon distribution $f(v)=\frac{k}{\bar{v}}(v / \bar{v})^{k-1} \exp \left[-(v / \bar{v})^{k}\right]$, in which, $f(v)$ stands for wind speed distribution function; $k$ is blade shape parameters, According to regulations, the general values can be1.8 2.8; $\bar{v}$ is the average wind velocity; $v(t)$ is the measured wind speed in real time. Assuming a microgrid, the relationship between wind generation power and wind speed is as the following formula ${ }^{[11]}$

$$
P(v)=\left\{\begin{array}{l}
0 \quad\left(v<v_{\mathrm{i}}, v>v_{\mathrm{o}}\right) \\
P_{\mathrm{w}} \frac{v-v_{\mathrm{i}}}{v_{\mathrm{o}}-v_{\mathrm{w}}} \quad\left(v_{\mathrm{i}}<v<v_{\mathrm{w}}\right) \\
P_{\mathrm{w}} \quad\left(v_{\mathrm{w}}<v<v_{\mathrm{o}}\right) \\
0 \quad\left(v>v_{\mathrm{o}}\right)
\end{array}\right.
$$

$P_{\mathrm{w}}(v)$ is rated generating power of the wind motor, also a certain value, $v_{\mathrm{i}}$ is start-up wind speed, $v_{\mathrm{W}}$ is rated wind speed, $v_{\mathrm{O}}$ is the blades that can withstand the maximum wind speed, from which it can be calculated $\sum_{j} C_{j}^{(2)} \Delta P_{j}[v(t)] \Delta t_{j}$. Considering load conditions, systematic energy loss is estimated and calculated on the right side of Equation (2), to check whether the system has reached the optimal configuration. Equation (2) varies with time and will not be satisfied automatically. If the value on the left is greater than that on the right, wind or light abandonment is considered. Instead, add power generation equipment ${ }^{[12]}$.

\subsection{Intelligent management system for wind and photovoltaic hybrid power}

\subsubsection{Intelligent controller}

Intelligent controller is controlling center of wind-solar complementary power generation system, which is composed of MCU, high-power MOSFET, contactor, circuit breaker, RS485 interface and wireless communication module, power conversion module and energy storage module, etc. The block diagram of a kind of intelligent management system is illustrated in Figure 4. 


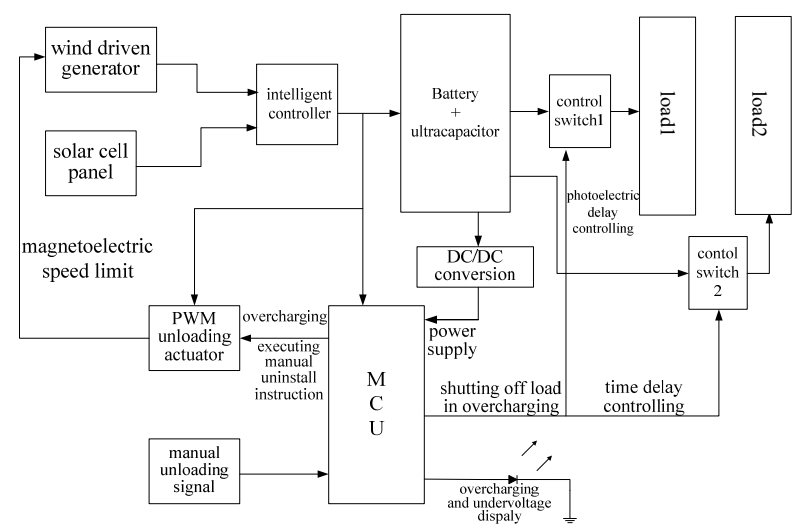

Fig 4. Block diagram of an intelligent management system of wind-solar complementary power generation

The intelligent controller converts and processes alternating current output from WTGS and solar photovoltaic panels, supplying power to loads of system. Excessive electric energy is stored in lithium ion battery module. Charging the module of WTGS is controlled by PWM stepless unloading. When both wind and solar energy are insufficient, the lithium ion battery module is controlled to supply power to load to prevent reverse charging from photovoltaic panels, the reverse connection aa well as opening of the lithium ion battery module. By network, working states of WTGS, solar photovoltaic panels and charge state of lithium ion battery modules can be monitored remotely. At present, grid-connection mode is popular for wind-solar complementary power generation systems with large capacity. Figure 5 is a schematic diagram of this kind of system.

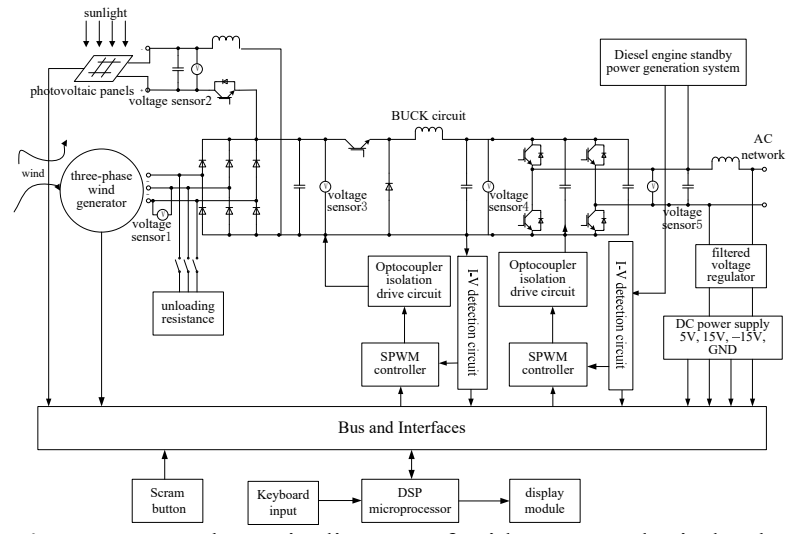

Fig 5. A schematic diagram of grid-connected wind-solar complementary power generation system

\subsubsection{Inverter}

$\mathrm{DC} / \mathrm{DC}$ module of inverter increases $48 \mathrm{~V}$ output voltage of lithium-ion battery module to $360 \mathrm{~V}$ through BOOST circuit. The main circuit of DC/AC inverter is composed of H-bridge IGBT circuit, fuse, filter, protection diode, etc. Adjustment of input voltage, output voltage, output frequency and output waveform by control and protection components, which is intent to prevent short circuit, overload, loss of voltage, overvoltage and etc. The inverted alternating current through sensors, states are back to intelligent management center, optimized processing, thus the electric energy that meets the requirements of grid connection is obtained.

\subsubsection{The intelligent management system}

It is composed of MCU, display module, keyboard and communication module, and etc. The charging module based on MOSFETs realizes double-standard three-stage charging of lithium-ion battery module, which drives IGBT to realize $\mathrm{DC} / \mathrm{AC}$ inverting, real-time protection, data reproduction and transmission, etc. At the same time, the experiment of magnetoelectric speed limiting of blade can be tried to put across in our laboratory. When wind speed is too high, a reverse magnetic resistance moment will generated to reduce the speed of blades and avoid damage.

\subsubsection{Storage of energy and on-line monitoring}

The energy storage system (such as lithium ion battery module) can be in state of charging, discharging and protection. On-line monitoring system monitors conditions of photovoltaic power generation unit, wind power generation unit, load and lithium-ion battery module simultaneously, entering the corresponding state under the corresponding conditions. In each state, in addition to completing its own work, it also provides corresponding display of technical parameters, communication between multiple systems and between the system and the upper computer according to user needs. An online monitoring block diagram of wind-solar complementary power generation system is shown in Fig. 6. ${ }^{[13]}$.

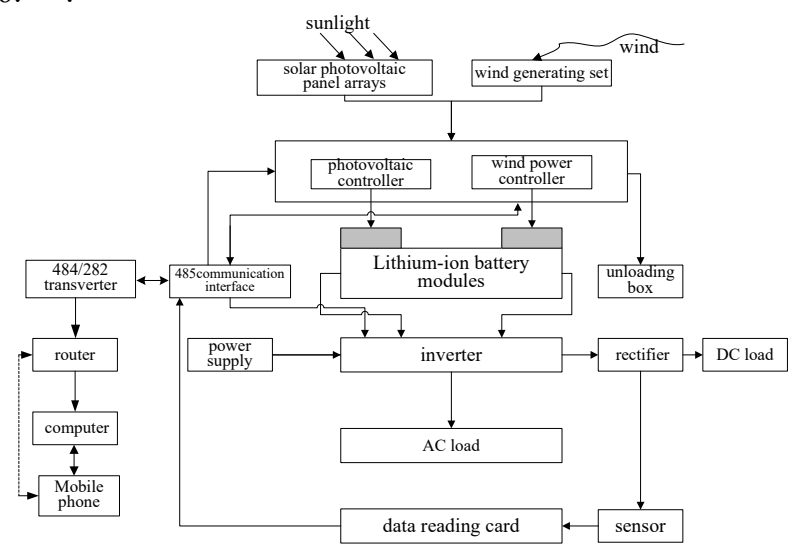

Fig 6. Block diagram of on-line monitoring of a windsolar complementary power generation system

Initializing and reading systematic parameters, such as photovoltaic array voltage, current, load, overvoltage, overcurrent protection parameters, magnetoelectric protection parameters, rated power, maximum power, output voltage, rated speed, starting wind speed, working wind speed, maximum restricted wind speed, diameter of blade, sweeping area, etc. While the technological parameters of lithium ion battery module is also obtained. Paying more attention to initialization of manmachine communication system and parameter setting of universal serial communication module are completed successively. Real-time sampling module, triggering signal from upper computer as well as control signal from user jointly estimate states of system. Firstly, sampling module collects real-time voltage, current and power to 
figure out conditions of photovoltaic array, wind power generation unit, lithium-ion battery module and load. Secondly, triggering signal from upper computer and controlling signal from user monitor the system jointly, or switch from one state to another. Lithium ion battery modules are charged by two-stage charging method with double standard. After a certain interval of time, the state parameters of photovoltaic array, wind power generation unit, lithium ion battery module and load are read online and displayed on screen or mobile phone end.

Monitoring state of lithium ion battery module, if the upper limit value is reached, standby lithium ion battery module + supercapacitor are used. Combination of the two can suppress impact of high frequency peak voltage and improve the utilization of resources ${ }^{[14]}$. During the power supply process for load, state of lithium-ion battery module should also be monitored to avoid overdischarge. When parameters of photovoltaic power generation unit, wind power generation unit, lithium ion battery module, load and other equipment inside the system reach the protective setting values, they enter protection state to avoid short-circuit, overvoltage, overcurrent and other possible hazards. These scenarios exist, for example, the magnetoelectric speed limit of wind motors, over-discharge of lithium ion battery module and over-voltage protection of load, etc. Communication module realizes communicating between system and upper computer. Convenient control of input, a variety of display output and flexible communication, are to ensure safe operation, also convenience for remote monitoring, maintenance, overhaul and management.

\section{Conclusions}

Complementary resources of solar and wind energy provide superior natural conditions for wind-solar complementary power generation. Technically and economically, photovoltaic power generation and wind power generation can be run simultaneously or separately, and technological improvement of wind power will reduce cost of power generation. DC and AC busbars facilitate entering and exiting of generating equipment or loads. Based on the law of energetic conservation, the proposed energy matching algorithm in this article provides a foundation for optimal systematic configuration, but economical factors have not been taken into account. At present, the wind-solar complementary power generation system has realized intelligent controlling, which can monitor operating conditions and state parameters of all equipment in real time.

\section{Corresponding author}

Dr. Prof. Wang Guangwei

School of Electronic Engineering, Tianjin Vocational and Technical Normal University, 1310 Dagu South Road, Hexi District, Tianjin, China

Zip code: 300222

Fax: (022)28181027

E-mail: wgw1633@163.com

\section{References}

1. Peng C., Zhang W. C. (2019) Review and prospect of wind-wind complementary power generation system. Journal of Guilin Institute of Aerospace Technology,2:208-214.

2. Fuller M. E. (2014) A battery model for constantpower discharge including rate effects. Energy Conversion \& Management, 88: 199-205.

3. Sheng J. (2020) Application of Phenolic Epoxy Vinyl Ester Resin Carbon Fiber Composites to Wind Turbine Blade. Technology and Education, 34(1):7-9.

4. Xu S., Tang H. (2021) Study on Optimal Capacity Allocation of wind and photovoltaic hybrid power in Ningxia Region. Electronic Test, 03:34-38.

5. Wang Z. L., Lu X., Zhuang M. H., et al. (2020) Study on spatial optimization of wind-wind complementary power generation system in northern China. Global Energy Internet, 3(1):97-104.

6. Yang D., Wang S.W, Wang Y., et al. (2019) Optimal allocation of extended photovoltaic complementary power generation capacity for grid-connected wind farms. Journal of Shandong University (Engineering Science), 49(5):44-51.

7. Li W., Tu L., Lin Y.G. (2013) Application Research of Hydraulic Technology in Wind Power Generation. Chinese Hydraulics \& Pneumatics, 03:1-9.

8. Hou Y. (2019) Remote Monitoring and Data Analysis of Wind-wind Complementary Power Generation System Based on GPRS. Journal of North China Institute of Aerospace Industry, 29(4):12-14.

9. Cheng C., Chen Z. H., SUN P. J., et al. (2021) Capacity optimization of wind-solar complementary system based on typical meteorological conditions. Acta Energiae Solaris Sinica, 42(2):110-114.

10. Yang Y., Yu T. Y., WANG D. W., et al. (2021) Prediction model of photovoltaic power based on NWP Kalman filter. Journal of Heilongjiang University of Science and Technology, pp.92-97.

11. Guan D. (2020) Research on Optimization of Load Forecasting Based on RNA-CS Algorithm. Zhuzhou, Hunan University of Technology Master Theses.122-128.

12. Yuan H. R. (2019) Study on power coordination control strategy of wind-wind complementary power generation system. Master thesis of Xinjiang University, Urumqi.

13. Guo Y. T, Wang J.N. (2019) Design of Online Fault Detection System for Wind-wind Complementary Circuits in Harsh Environment. Journal of power supply, 17(6):137-144.

14. Yu D. X.(2018) Study on capacity configuration and inverter of wind-wind complementary power generation system. Master thesis of Yangzhou University. pp.132-147. 